\title{
HIV Estimation and Projection in Sri Lanka (2019-2025); Application of AIDS Epidemic Model and Spectrum
}

\author{
Suchira Suranga, Ariyaratne Manathunge, Wiwat Peerapatanapokin
}

\begin{abstract}
Introduction: Since the beginning of the HIV epidemic in the late 1980s, Sri Lanka has been classified as a country with low level HIV epidemic. Even among key populations, HIV prevalence has not exceeded beyond 5\%. HIV estimation and projection in Sri Lanka are more important than ever when the country is trying to reach ending AIDS epidemic targets by 2025.

Objective: This study aims to generate HIV estimates for Sri Lanka and identify the pattern of the AIDS epidemic and develop future directions.

Method: AIDS Epidemic Model (AEM) and Spectrum were used as tools to generate the estimates by Technical Working Group (TWG) which was represented by the relevant government, nongovernment, UN and technical partners. Sub-national estimates were not attempted during the current AIDS Epidemic Modelling due to the lack of region-specific data as well as expected low numbers in a sub-national estimate in Sri Lanka.

Results: Results of national estimates indicate that there are 3,600 people living with HIV (PLHIV) in Sri Lanka in 2019. Of this estimate, 3,550 are adults who are 15 years and older, and the male to female ratio was 2.93. The trend of PLHIV is relatively stable in the past five years implying the state of balance between new infections and AIDS related deaths. Total new HIV infections were estimated at 140 in 2019 with 137 new HIV infections among adults 15 years and older. Although the epidemic is declining in terms of numbers, new HIV infections among men who have sex with men (MSM) as share of total new HIV infections is growing. In 2019, 169 AIDS-related deaths are estimated among adults living with HIV.
\end{abstract}

Conclusions: AIDS epidemic in Sri Lanka is seems to be under control but not yet over. The National Strategic Plan scenario showed that ending AIDS is achievable. However, this needs scale up of current HIV prevention efforts based on the informed policy and decision making.

Key words: HIV estimation and Projection, AIDS Epidemic Model, Spectrum, New Infections, Estimated AIDS Related Deaths

\footnotetext{
Authors: corresponding author; ${ }^{11}$ M. Suchira Suranga, B.SC (Agric.), M.Sc (Bio-Statistics), M.Sc (Management), P.Phil (SRH), Director (Organizational Learning and Evaluation), FPA Sri Lanka, Colombo. (D) https://orcid.org/0000-0002-8200-9572, email:- suchirasuranga@gmail.com

${ }^{2}$ Dr. Ariyaratne Manathunge, MBBS, MSC, MD, Consultant Venereologist, National STD/AIDS Control Programme, Colombo. Email: ariyaratne1@gmail.com, (iDhttps://orcid.org/0000-0001-9514-9227

${ }^{3}$ Dr. Wiwat Peerapatanapokin, MD, PhD (Epidemiology), Non-Resident Senior Fellow, East-West Center, Email: wiwat@hawaii.edu
}

Conflict of interest: Authors claim no conflicts of interest Funding: Global fund and WHO country office, Sri Lanka 


\section{Full article}

\section{Introduction}

Sri Lanka has been categorized as a country with low-level of HIV epidemic and the HIV prevalence has not consistently exceeded $5 \%$ in any of the high risk sub-populations such as female sex workers (FSW), men who have sex with men (MSM), beach boys (BB) and people who inject drugs (PWID). ${ }^{(1)}$ However, Sri Lanka has adopted the SDG target of "End AIDS by 2030" and has accepted the challenge of achieving this target five years before the rest of the world, i.e. by 2025 . In this journey through the fast track, the National STD/AIDS Control Programme is the pioneer government institution in Sri Lanka which is taking the leadership and decisions to guide the national response to HIV and reach this goal timely. ${ }^{(2)}$

The total number of PLHIV diagnosed and alive is 2,709 (cumulative reported number minus cumulative reported deaths). However, these are cumulative figures since 1987, and there are deaths that are not reported as AIDS deaths. Out of the total 1,656 PLHIV who are currently linked with HIV treatment and care services, 1,574 have been started on antiretrovirals (ART), and 1,338 were having viral suppression. ${ }^{(2)} A$ total of 350 HIV infected persons were newly reported during 2018. This is an increase of $23 \%$ from the number reported during 2017. Analysis of probable mode of HIV transmission in 2018 shows that the male-to-male (46\%) and male-to-female sexual transmission $(41 \%)$ are the most frequent modes of transmission. Of these, male-to-male HIV transmission has become the predominant mode of HIV transmission since 2017. Very low rates of HIV transmission due to injecting drug use and mother-to-child HIV transmission were reported.

Sri Lanka has identified different high risk subpopulations for HIV prevention interventions such as female sex workers (FSW), men who have sex with men (MSM), beach boys (BB), clients of sex workers and drug users (DU) as most-at-risk populations
(MARPs). ${ }^{(3)}$ HIV prevalence estimation carried out in the Integrated Biological and Behavioural Survey (IBBS) showed that HIV prevalence among FSW, MSM and BB were $0.2 \%$ while the HIV prevalence among injecting drug users was $0 \%$. ${ }^{(4)}$ It is noteworthy to highlight that for the first time in history, transgender women (TGW) were included in IBBS and HIV prevalence was found to be higher (1.2\%) than other key populations. National size estimation study carried out in 2017/2018 showed an estimate of 30,000 female sex workers, 40,000 MSM, 4,500 BBs, 900 PWID and 2,200 TGW in the country. ${ }^{(5)}$

New HIV infections and people living with HIV (PLHIV) in Sri Lanka had been estimated using Estimation and Projection Package (EPP) from 2009 to 2016. During 2017, the National estimation working group decided to use the AIDS epidemic model (AEM) in place of EPP as this would provide more details such as building intervention scenarios and impact analysis, etc. NSACP conducted a workshop to adopt AIDS Epidemic Model in Sri Lankan context in Bangkok, Thailand in October 2017. The initial model developed in 2017 was improved gradually over the years. The latest HIV estimation process was conducted from $24^{\text {th }}$ to $27^{\text {th }}$ February 2020 in Colombo, Sri Lanka with the technical assistance from the East West Center in USA. This report describes the process and the results of PLHIV estimation done in 2020 using AEM and Spectrum in Sri Lanka.

\section{Objectives}

The HIV estimation process was conducted with the aim of generating HIV estimates for Sri Lanka for the year 2019 and to identify the pattern of the AIDS epidemic and to develop future directions.

\section{Methods}

A consultative process with more stakeholder engagement was adopted for development and finalization PLHIV estimation in Sri Lankan context. A wider range of stakeholder 
including relevant staff of NSACP, and peripheral STD clinics, M\&E officers from the Family Planning Association of Sri Lanka participated in the estimation process using AEM and Spectrum. The team, together with a technical assistant from the East West Center, collected, generated, compared, examined, and built consensus on the data inputs for the AEM workbooks. The consultative meeting started with a comprehensive introduction to help the team understand the technical aspects of the AEM software, data needs, critical issues, assumptions, and validating results based on the context of the epidemic in Sri Lanka.

\section{AIDS Epidemic Model}

The AIDS Epidemic Model (AEM) is a process model which considers the behavioural and biological processes that transmit HIV. ${ }^{(6)}$ Using trends in risk behaviors among the important sub-populations in the particular country or region as inputs, the model determines the HIV transmission probabilities necessary to fit observed epidemiological patterns, as seen in surveillance data. It then calculates the number of new infections through key routes of transmission including marital sex, extramarital (casual) sex, sex work, male-male sex, needle sharing, and mother-to-child transmission (Figure 01). Specific outputs of the model include the number of new, current and cumulative HIV infections and AIDS related deaths for each year. ${ }^{(7)}$ In contrast to the curve fitting approaches used in the UNAIDS workbook method and the Estimation and Projection Package (EPP), the Asian Epidemic Model (AEM) is a full process model that mathematically replicates the key processes driving HIV transmission in Asia. ${ }^{(8)}$

AEM is based on the epidemiological patterns commonly observed in concentrated epidemics, where the bulk of HIV transmission occurs among key populations and their sexual partners including, 1) Men who have sex with men (MSM) and male sex workers (MSW), 2) Female sex workers (FSW) and their clients, 3) People who inject drugs (PWID, male and female), 4) Transgender populations (TG), 5)
Lower-risk members of the general population (both male and female). Each of these populations and their primary behaviours which transmit HIV, including vaginal sex, anal sex, and sharing of injecting equipment are included in the model by the working group. The key inputs includes but not limited to, 1) The size of these key populations, 2) The average duration for which people remain in key populations, 3) The frequency of risk behaviours, 4) The levels of protective measures taken with different partner types, e.g., condom use between sex workers and clients, reducing the fraction of injections shared or the prevalence of sharing, 5) HIV and STI prevalence in each key affected population. (6)

Figure 01: HIV transmission dynamics in AEM

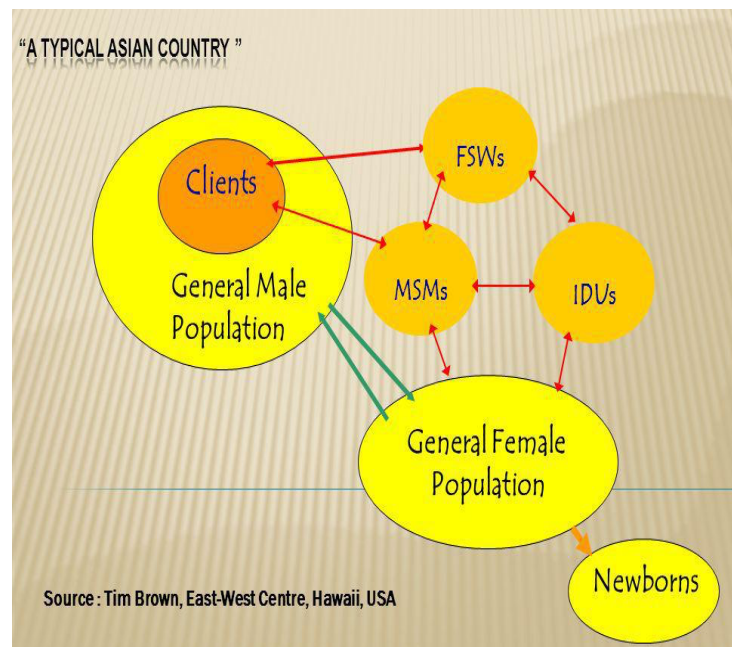

\section{Spectrum Estimates}

As AEM is an HIV estimation model for adults defined as age 15 and above.) To generate national estimates including children (0-14 years old) and components of mother-to-child transmission of HIV (MTCT), incidence trend from AEM model is imported into Spectrum software to generate estimates for all people living with HIV including children and pregnant women. Spectrum software provides the option to choose and import the AEM baseline workbook that reads in the spectrum and generates estimates for both adults and children (Figure 02). Once the AEM workbook is finalized, the AEM outputs were imported 
into the spectrum software (Version 5.87 used for the current estimation process). The adult demographic and adult ART inputs are checked to be consistent between AEM and Spectrum. The modeling results from both models were inspected and calibrated with regards to comparability values related to key estimates of AEM and Spectrum. After certain adjustments, the number of adult PLHIV was consistent with that of AEM estimates.

\section{Scope and limitations}

This paper focuses on the processes, key findings, program implications, and recommendations based on the results generated by AEM and Spectrum. Technical descriptions and instructions on how to use the AEM software is not described in this article. However; such information can be found in the 2014 AEM 4.02b manual "Assessing HIV Program Impacts with AIDS Epidemic Model (AEM) - A Tutorial Introduction to the AEM Suite of Tools and Workbooks" developed by the EWC research program in Hawaii, USA. It was observed that the AEM tool does not have age disaggregation to describe the projections and interventions for different age groups. The AEM Included FSW, MSM, and PWID as key population. Further details can be obtained from the AEM technical report, 2020. ${ }^{(9)}$
Figure 02: AEM - Spectrum Structure

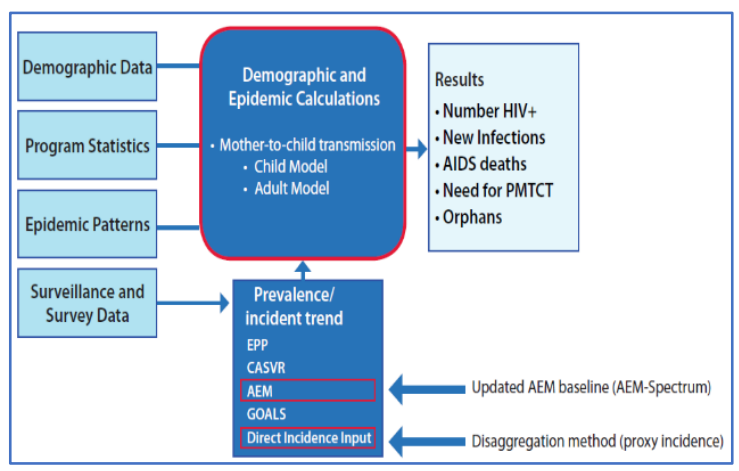

\section{Results}

Table 01 provides a summary of outputs generated by AEM (baseline scenario) and spectrum software. Estimated annual new infections among adults in 2019 is 137 and then gradually reduce to 113 by 2025 . However, the estimated number of deaths due to AIDS in 2019 is 169 and gradually increase to 176 by 2025 . The estimated number of adult PLHIV are decreasing over time from 3,550 in 2009 to 3,231 in 2025. The epidemic is transforming to a more and more male type epidemic which is due to more HIV transmission among MSM. Spectrum estimates provide HIV estimates among both adults and children. Total estimated number of PLHIV including children is 3,605 in 2019 and expected to be reduced up to 3,383 by 2025 .

\section{HIV new infections among adults by Key Populations and their partners}

Table 1: Summary of AEM and Spectrum outputs in baseline scenario (2019 - 2025)

\begin{tabular}{lrrrrrrr}
\hline & $\mathbf{2 0 1 9}$ & $\mathbf{2 0 2 0}$ & $\mathbf{2 0 2 1}$ & $\mathbf{2 0 2 2}$ & $\mathbf{2 0 2 3}$ & $\mathbf{2 0 2 4}$ & $\mathbf{2 0 2 5}$ \\
\hline AEM Results (Excluding Children) & & & & & & & \\
\hline New HIV infections: & 137 & 124 & 121 & 119 & 117 & 115 & 113 \\
\hline Current PLHIV: & 3,550 & 3,520 & 3,475 & 3,419 & 3,358 & 3,294 & 3,231 \\
\hline Annual death: & 169 & 155 & 166 & 175 & 178 & 178 & 176 \\
\hline Annual ART needs: & 3,550 & 3,520 & 3,475 & 3,419 & 3,358 & 3,294 & 3,231 \\
\hline Number on ART: & 1,668 & 1,776 & 1,775 & 1,772 & 1,767 & 1,760 & 1,752 \\
\hline Male-Female Inc Ratio: & 4.15 & 4.61 & 4.70 & 4.77 & 4.87 & 4.99 & 5.12 \\
\hline Cumulative infections: & 8,106 & 8,230 & 8,351 & 8,470 & 8,587 & 8,702 & 8,815 \\
\hline Cumulative deaths: & 4,556 & 4,711 & 4,877 & 5,052 & 5,230 & 5,408 & 5,584 \\
\hline Cumulative M/F Ratio: & 2.93 & 2.94 & 2.96 & 2.98 & 3.00 & 3.01 & 3.03 \\
\hline
\end{tabular}


Table 1 cont

\begin{tabular}{lrrrrrrr}
\hline & $\mathbf{2 0 1 9}$ & $\mathbf{2 0 2 0}$ & $\mathbf{2 0 2 1}$ & $\mathbf{2 0 2 2}$ & $\mathbf{2 0 2 3}$ & $\mathbf{2 0 2 4}$ & $\mathbf{2 0 2 5}$ \\
\hline Spectrum Results (Including Children) & & & & & & \\
\hline New HIV infections (Total) & 140 & 126 & 124 & 121 & 119 & 116 & 114 \\
\hline New HIV infections (Male) & 112 & 103 & 101 & 99 & 98 & 96 & 94 \\
\hline New HIV infections (Female) & 28 & 23 & 23 & 22 & 21 & 20 & 19 \\
\hline Current PLHIV (Total) & 3,605 & 3,595 & 3,578 & 3,543 & 3,496 & 3,441 & 3,383 \\
\hline Current PLHIV (Male) & 2,565 & 2,570 & 2,568 & 2,556 & 2,535 & 2,508 & 2,479 \\
\hline Current PLHIV (Female) & 1,040 & 1,026 & 1,010 & 987 & 961 & 933 & 904 \\
\hline HIV Prevalence (15-49) & 0.03 & 0.02 & 0.02 & 0.02 & 0.02 & 0.02 & 0.02 \\
\hline Annual AIDS death (Total) & 135 & 103 & 108 & 124 & 134 & 138 & 140 \\
\hline Annual AIDS death (Male) & 96 & 71 & 75 & 85 & 92 & 95 & 97 \\
\hline Annual AIDS death (Female) & 39 & 32 & 33 & 39 & 42 & 43 & 43 \\
\hline
\end{tabular}

Table 2: Number of new infections in 2019; Mode-of-Transfer among different sub-populations

\begin{tabular}{|c|c|c|c|c|c|c|c|c|}
\hline & & \multicolumn{6}{|c|}{ New Infections Occurring Among } & \multirow[b]{2}{*}{ Total (\%) } \\
\hline & & $\begin{array}{r}\text { SW } \\
\text { Clients }\end{array}$ & $\begin{array}{l}\text { Male } \\
\text { PWID }\end{array}$ & MSM & $\begin{array}{r}\text { LR } \\
\text { Males }\end{array}$ & FSW & $\begin{array}{r}\text { LR } \\
\text { Females }\end{array}$ & \\
\hline \multirow{6}{*}{ 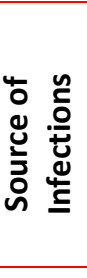 } & Sex with Female sex workers & 28 & & & & & & 28 \\
\hline & Spouses/regular partners & & & & 3 & & 14 & 18 \\
\hline & Casual sex partners & & & & & & 2 & 2 \\
\hline & Injecting drug use & & 7 & & & & & 7 \\
\hline & Sex with MSM & & & 72 & & & 4 & 77 \\
\hline & Sex with clients of FSW & & & & & 6 & & 6 \\
\hline & Total & 28 & 7 & 72 & 3 & 6 & 20 & 137 \\
\hline
\end{tabular}

As per the AEM results (table 2), 137 new infections are estimated in 2019. More than half of new infections (53\%) estimated among MSM followed by clients of FSW (21\%) and low risk women (15\%). The estimated number of new infections among male PWID (5\%), FSW (4\%) and low risk males (2\%) are extremely low. Percentages of new infections among MSM are increasing over time. Sri Lanka has been implemented interventions among FSW for more than two decades. Proportion of new infections among FSW and clients of FSW showing a reducing trend over time which can be attributed to a high level of condom use among FSW. However, the number of infections among low risk females is increasing during the next few years reflecting HIV transmission from MSM who have sex with female partners. These results clearly indicate that Sri Lankan HIV prevention programme needs to be more focused on designing interventions for MSM. Although there is no considerable drive by the PWID, the numbers of infections among PWID are estimated to increase slightly over the next few years.

\section{Estimated number adults living with HIV by Key Populations}

AEM estimated 3,550 adult PLHIV in the country and out of them 1,439 (41\%) are high risk males. High risk males include clients of FSW (500), MSM (924) and male PWID (15). The number of high-risk females among PLHIV considered to be significantly low (4\%) which includes 134 HIV positive FSW. Around one third of the PLHIV (31\%) considered to be low risk males and balance $25 \%$ consists of low risk females. It is noteworthy that although the HIV prevalence is relatively low (e.g. male $=0.02 \%$ and female $=0.01 \%$ ), almost $56 \%$ of the total new PLHIV coming from low risk population as the size of low risk population is very high. However, it is important to highlight that the proportion of high risk PLHIV is showing an increasing trend over the next decade with an increase of new infections among PWID and MSM. 


\section{Probable mode of transmission among adults}

Out of the 137 estimated new infections in 2019, almost half of infections (53\%) are transmitted through male-to-male sex, followed by sex work (25\%), husband-to-wife (14\%), wife-to-husband (2\%) and casual sex (1\%). Only 5\% transferred through injecting drug use. As illustrated in table 8 , the proportion of male-to-male transition is estimated to be increasing up to $64 \%$ by the end of 2025.

However, the proportion of HIV transmission through sex work shows a decreasing trend over time due to expected increase in condom use among FSWs. As per the results of AEM, we are not expecting a significant change of proportion in HIV transmission through regular sexual acts among spouses, casual sex and injecting drug use. However, the estimated proportion of sub-population among 3,550 people living with HIV (in 2019) shows a different picture. The majority (31\%) of PLHIV are low risk males followed by MSM (26\%), low risk female (25\%), clients of FSW (14\%) and FSW (4\%). The percentage of male PWID estimated to be almost zero. Most of low risk male PLHIV were infected in the past when they were in high risk group at that time. Most of low risk female PLHIV were infected from their spouses who were in high risk or ex-high risk groups.

\section{AEM national strategic plan scenario}

As described so far, AEM results in the baseline scenario indicated that country cannot achieve 90:90:90 targets by 2025 or 2030 if there are no additional interventions during the next few years. Therefore, the country team worked on the national strategic plan scenario to compare the estimates, if we implanted the national strategic plan (2019-2022) and ensure the expected results in ART coverage and behavioral indicators. Condom use among FSW and reachable MSM (MSM-1) was gradually increased by up to $90 \%$ by the end of 2022 and STI prevalence among MSM and FSW was decreased to $4.8 \%$ and $4 \%$ respectively. Condom use of unreachable MSM (MSM-2) also increased up to $80 \%$ by end of 2022 . Condom use among HIV sero-discordant couples was increased by up to $68 \%$ by the end of 2022. The percentage of male PWID who share needles is decreased to $27 \%$ by the end of the strategic plan period. Percent of all injections shared (among those who share) also gradually reduced from $90 \%$ in 2019 to $41 \%$ by 2022 . Expected ART coverage was increased by up to $95 \%$ by the end of 2022 . All other inputs were similar to the baseline scenario. AEM generated impressive results when applying the national strategic plan scenario (Table 3).

Figure 3: Percentage of new infections by mode-of-transmission 2019-2025

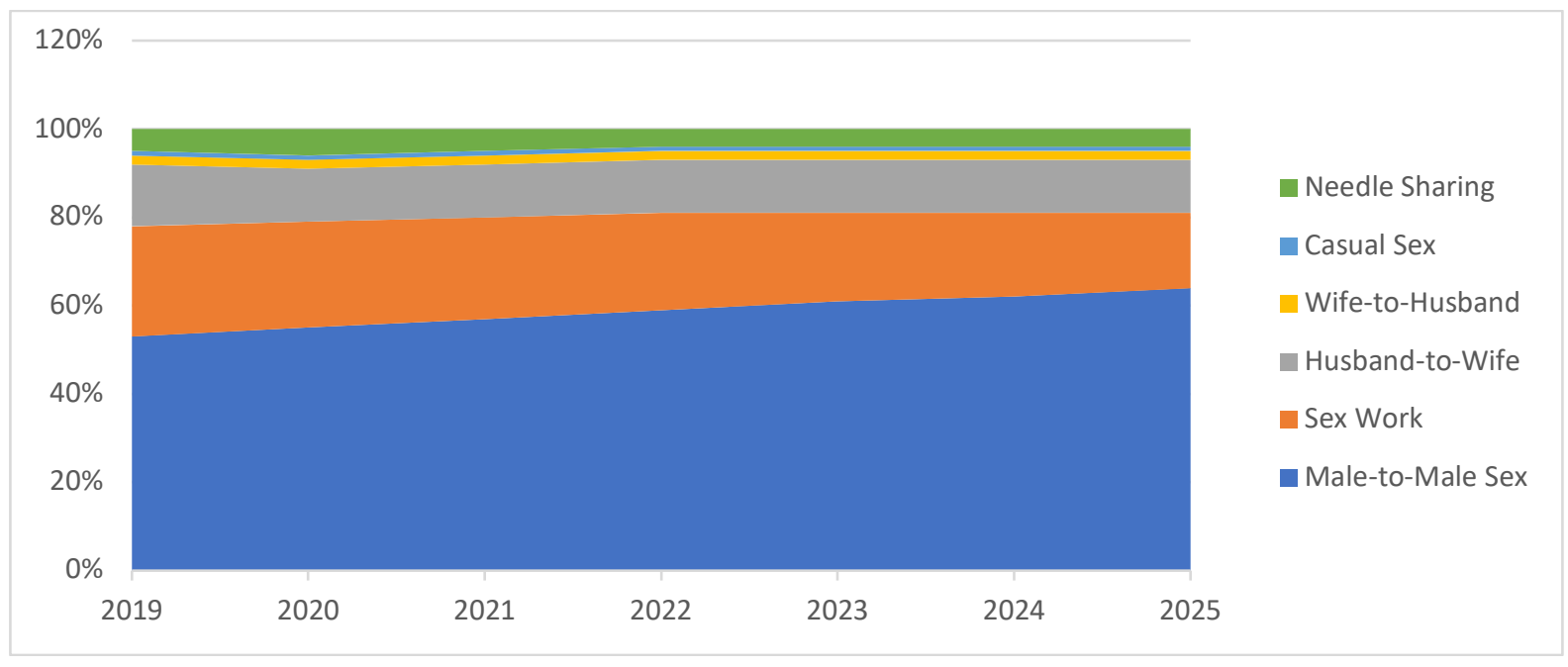


Table 3: Summary of estimates under national strategic plan scenario (2019-2025)

\begin{tabular}{lrrrrrrr}
\hline & $\mathbf{2 0 1 9}$ & $\mathbf{2 0 2 0}$ & $\mathbf{2 0 2 1}$ & $\mathbf{2 0 2 2}$ & $\mathbf{2 0 2 3}$ & $\mathbf{2 0 2 4}$ & $\mathbf{2 0 2 5}$ \\
\hline New HIV infections (BS) & 137 & 124 & 121 & 119 & 117 & 115 & 113 \\
\hline New infections (NSP) & $\mathbf{1 3 7}$ & $\mathbf{1 2 3}$ & $\mathbf{8 4}$ & $\mathbf{5 0}$ & $\mathbf{2 7}$ & $\mathbf{2 5}$ & $\mathbf{2 3}$ \\
\hline Current PLHIV (BS) & 3,550 & 3,520 & 3,475 & 3,419 & 3,358 & 3,294 & 3,231 \\
\hline Current PLHIV (NSP) & $\mathbf{3 , 5 5 0}$ & $\mathbf{3 , 5 2 2}$ & $\mathbf{3 , 4 7 9}$ & $\mathbf{3 , 4 3 0}$ & $\mathbf{3 , 3 7 6}$ & $\mathbf{3 , 3 2 4}$ & $\mathbf{3 , 2 7 1}$ \\
\hline Annual deaths (BS) & 169 & 155 & 166 & 175 & 178 & 178 & 176 \\
\hline Annual death (NSP) & $\mathbf{1 6 9}$ & $\mathbf{1 5 1}$ & $\mathbf{1 2 7}$ & $\mathbf{9 9}$ & $\mathbf{8 2}$ & $\mathbf{7 7}$ & $\mathbf{7 6}$ \\
\hline Annual ART need (BS) & 3,552 & 3,521 & 3,476 & 3,420 & 3,359 & 3,295 & 3,232 \\
\hline Annual ART need (NSP) & $\mathbf{3 , 5 5 2}$ & $\mathbf{3 , 5 2 4}$ & $\mathbf{3 , 4 8 1}$ & $\mathbf{3 , 4 3 2}$ & $\mathbf{3 , 3 7 7}$ & $\mathbf{3 , 3 2 5}$ & $\mathbf{3 , 2 7 2}$ \\
\hline Number on ART (BS) & 1,668 & 1,776 & 1,775 & 1,772 & 1,767 & 1,760 & 1,752 \\
\hline Number on ART (NSP) & $\mathbf{1 , 6 6 8}$ & $\mathbf{1 , 9 4 2}$ & $\mathbf{2 , 3 5 0}$ & $\mathbf{2 , 8 3 2}$ & $\mathbf{3 , 0 6 8}$ & $\mathbf{3 , 0 7 6}$ & $\mathbf{3 , 0 8 1}$ \\
\hline Male-Female Infection Ratio (BS) & 4.15 & 4.61 & 4.70 & 4.77 & 4.87 & 4.99 & 5.12 \\
\hline Male-Female infection Ratio (NSP) & $\mathbf{4 . 1 5}$ & $\mathbf{4 . 6 0}$ & $\mathbf{4 . 2 4}$ & $\mathbf{3 . 7 7}$ & $\mathbf{3 . 2 3}$ & $\mathbf{3 . 2 1}$ & $\mathbf{3 . 1 8}$ \\
\hline Note:- BS B Baseline sCenariO & NSP Nati \\
\hline
\end{tabular}

Note :- $\mathrm{BS}=$ Baseline scenario,

NSP $=$ National Strategic Plan scenario

As given in table 3 , the NSP scenario generated favourable estimates in terms of ending AIDS by 2025. Number of new infections by the end of 2025 reduced to 23 as opposed to 113 new infections generated in the baseline scenario. Estimated annual AIDS related death in 2025 also reduced from 176 in baseline scenario to 76 in NSP scenario with the increase of ART coverage. The estimated number of PLHIV on ART increased up to 3,081 by end of 2025. New infections among MSM drastically reduced and therefore, male: female infection ratio reduced from 5.12 (baseline scenario) to 3.18 (NSP scenario).

\section{Discussion}

Sri Lanka is still a low-level HIV epidemic country with an adult HIV prevalence of less than $0.1 \%$. The major mode of transmission in the past was from commercial sex work. The current major mode of HIV transmission is MSM. The future major mode of transmission also will be MSM. Sri Lanka has not seen a major PWID epidemic. The contribution of MSM is getting higher and higher. Since MSM is the major mode of transmission currently as well as in the future, increasing prevention and treatment among MSM is the key to the future. However, maintaining the success in the FSW program is necessary.

Figure 4 : New infections among adults, 2010-2030, baseline vs NSP scenarios

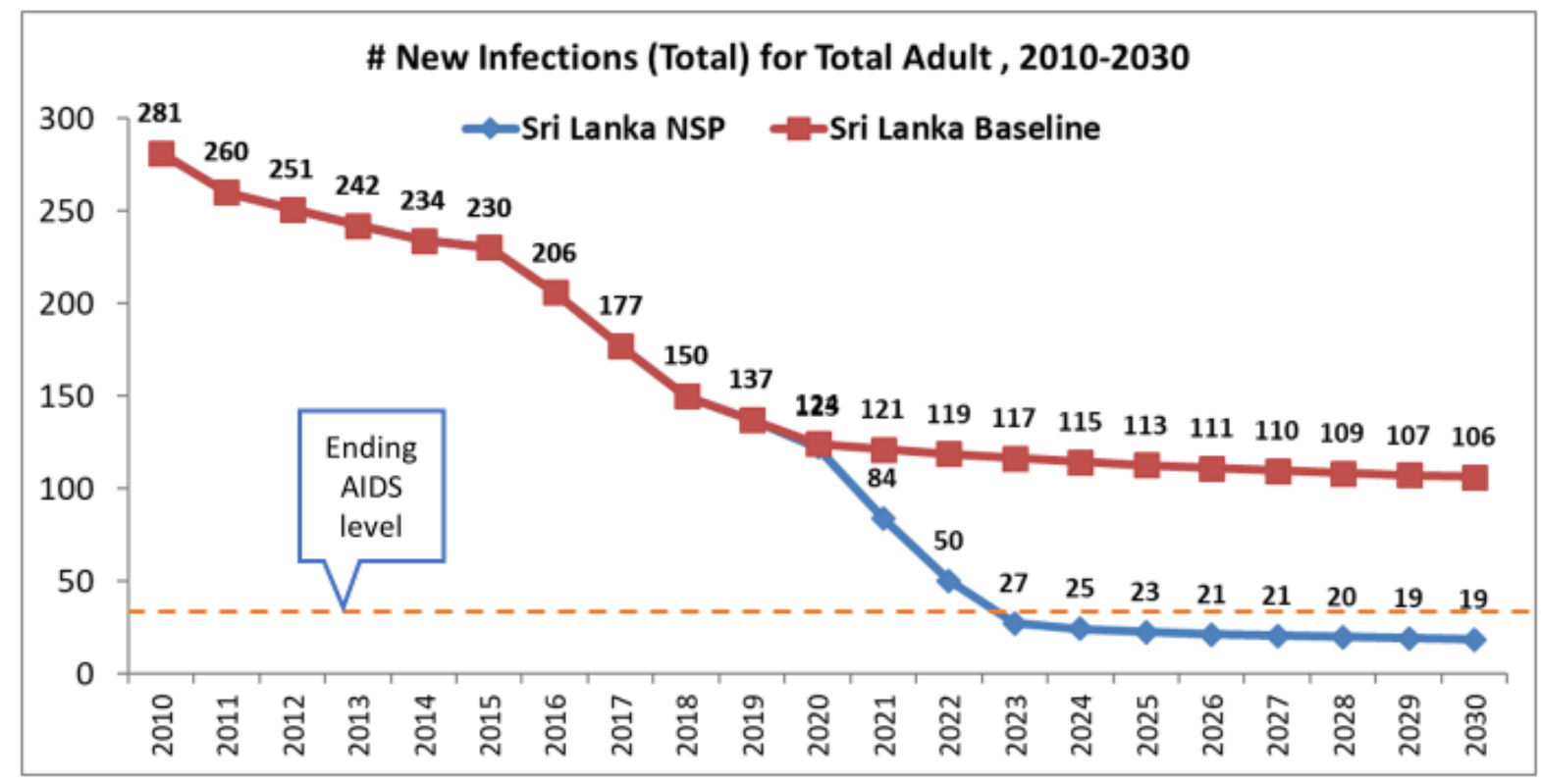


Baseline scenarios show continuing to reduce the HIV epidemic, but not enough to end AIDS by 2025. National Strategic scenarios can produce ending AIDS impact by 2025 (New infections in 2010 is 280 while new infections from NSP scenarios will be 23 in 2025 which is more than $90 \%$ reduction from the year 2010).

ART Coverage is around $50 \%$. The main reason for this low ART coverage is the low number of PLHIV who know their status (only 64\% in 2019). To increase this level (first 90 target), Sri Lanka needs to increase targeted HIV testing. Higher ART coverage will help to reduce all kinds of transmission especially reducing transmission between husband and wife. Higher ART coverage will reduce AIDS related deaths significantly.

\section{Conclusions}

Sri Lanka is still a low-level HIV epidemic country with an adult HIV prevalence of less than $0.1 \%$. AIDS epidemic in Sri Lanka is seems to be under control but not yet over. The National Strategic Plan scenario showed that ending AIDS is achievable. However, this needs scale up of current HIV prevention efforts based on the informed policy and decision making

\section{References}

1. UNAIDS/WHO. Guidelines for second generation HIV surveillance. World Health Organization. Geneva : s.n., 2000.

2. NSACP. Annual Report - 2018. Colombo, Sri Lanka : National STD AIDS Control Programme, Sri Lanka, 2019.

3. Sri Lanka National HIV Prevention Strategic Plan; 2018-2022. Colombo, Sri Lanka : National STD AIDS Control Programme, 2017.

4. Integrated Biological and Behavioral Surveillance Survey - 2018. Colombo, Sri Lanka : National STD AIDS Control Programme, 2018.

5. National Size Estimation for the Most at Risk Population for HIV in Sri Lanka. Colombo, Sri Lanka : National STD AIDS Control Programme, 2018.

6. Siripong, Nalyn, et al. Assessing HIV Program Impacts with the AIDS Epidemic Model (AEM). Honolulu, Hawaii : East-West Center Research Program, 2016.

7. Family Health International. The Asian Epidemic Model (AEM); Projections for HIV/AIDS in Thailand
(2005-2025). Bangkok, Thailand : Ministry of Public Health, Thailand, 2008. ISBN: 978-974-314-141-1.

8. The Asian Epidemic Model: A process model for exploring HIV policy and programme alternatives in Asia. Brown, T and Peerapatanapokin, W. 1, Sexually Transmitted Infections, Vol. 80, pp. 19-24.

9. Suranga, Suchira and Manatunge, Ariyaratne. Technical Report on HIV Estimation in Sri Lanka 2019. Colombo, Sri Lanka : Sri Lanka College of Sexual Health and HIV Medicine, 2020. 